\title{
Impacto del Plan Garantizado de Salud con prima comunitaria única en la demanda del seguro privado de salud en Chile
}

\author{
Eduardo Bitran, Fabián Duarte, \\ Dalila Fernandes y Marcelo Villena
}

\section{Resumen}

En 2012 se envió al Congreso de Chile un Plan Garantizado de Salud para el sistema privado de salud, diseñado para ofrecer un plan de características estandarizadas y tarifa plana. En este trabajo se evalúa el impacto que tendría en la demanda de seguros de salud la introducción de este plan. Los resultados sugieren que la cartera del seguro privado disminuiría un 12,39\%, lo que significa que alrededor de 400.000 personas se cambiarían al sistema público, exacerbando el problema de selección adversa que enfrenta el sistema y acarreando un pasivo fiscal de 200 millones de dólares anuales.

\section{Palabras clave}

Salud, sector privado, seguro de enfermedad, precios, oferta y demanda, servicios de salud, sector público, datos estadísticos, Chile

\section{Clasificación JEL}

1130, C250

\section{Autores}

Eduardo Bitran es Vicepresidente Ejecutivo de la Corporación de Fomento de la Producción (CORFO). Correo electrónico: eduardo.bitran@uai.cl.

Fabián Duarte es Profesor Asistente del Departamento de Economía de la Facultad de Economía y Negocios de la Universidad de Chile. Correo electrónico: fabduarte@ fen.uchile.cl.

Dalila Fernandes es Gerente de Cuentas de App Annie. Correo electrónico: dalilafernandes@gmail.com.

Marcelo Villena es Vicedecano de la Facultad de Ingeniería y Ciencias de la Universidad Adolfo Ibáñez (UAl) de Chile. Correo electrónico: marcelo.villena@uai.cl. 



\section{Introducción}

La experiencia chilena en la provisión de seguros de salud es bastante particular. Primero, pocos países ofrecen la opción de elegir entre múltiples planes de seguros de salud, como en el caso chileno. Segundo, el sistema chileno presenta una segmentación estructural, con una población de bajos ingresos y alto riesgo acogida al seguro público, el Fondo Nacional de Salud (FONASA), y una población de altos ingresos y de bajo riesgo, que por lo general acude a un seguro privado, las instituciones de salud previsional (isapres), denotando un gran problema de selección adversa. A partir de 2004, se introdujo un régimen general de garantías de salud, las Garantías Explícitas en Salud (GES), pionero en América Latina, con el objetivo de establecer derechos de atención a los pacientes y así procurar una mayor equidad en el sistema (véase Missoni y Solimano, 2010). A pesar de dichas reformas, el sistema continuó acusando evidencias de selección adversa por parte de la demanda.

En este contexto, la Comisión de Reforma de la Salud (CRS), establecida por el Poder Ejecutivo en 2011, propuso un nuevo Plan Garantizado de Salud (PGS), diseñado para ofrecer a todos los usuarios del sistema privado un plan de características estandarizadas y tarifa plana, que se presentó al Congreso Nacional en 2012. La prima de este nuevo plan, que no depende de la edad o el género, se cobra a cada integrante del grupo familiar (cotizante y dependiente) y debe ser financiada con el 7\% de cotización obligatoria del individuo. En caso de que se generen excedentes respecto a la cotización obligatoria, el afiliado podrá usar la diferencia para contratar un seguro complementario en la isapre en la que cotiza, el que sí estará sujeto al uso de una tabla de factores de riesgo. En caso de que la cotización obligatoria sea insuficiente, el afiliado deberá aportar una cotización adicional para cubrir la diferencia ${ }^{1}$.

Según estimaciones de la CRS, se prevé un valor del PGS de hasta 21.000 pesos chilenos para 2011, suponiendo una cobertura del 50\% del arancel de la Modalidad Libre Elección (MLE) del nivel 1 del FONASA ${ }^{2}$. Sin embargo, la negociación en el Senado ha modificado los copagos, reduciéndolos significativamente, lo cual debería presionar el precio del PGS al alza.

EI PGS reúne los beneficios ya determinados en las Garantías Explicitas de Salud (GES), la Cobertura Adicional para Enfermedades Catastróficas (CAEC) y, además, un grupo de enfermedades no cubiertas en el plan GES, para las cuales se definirá una red de prestadores y un nivel de bonificaciones que deben seguir, por lo menos, las especificaciones del arancel de la Modalidad Libre Elección del nivel 1 del seguro público FONASA. Además, en el PGS se eliminarán las carencias y preexistencias para los afiliados del sistema privado que desean contratar el seguro de salud dentro del sistema de isapres. Esta medida permite que los individuos ya afiliados al sistema privado se muevan sin restricciones entre las distintas aseguradoras. Sin duda, esto ayudaría a mitigar el problema de los afiliados que se vuelven cautivos de una isapre cuando alcanzan una determinada edad o desarrollan alguna enfermedad crónica. El PGS, al eliminar las carencias y preexistencias dentro del sistema privado y considerar un fondo de compensación de riesgos que permite compensar a los planes de isapre que tienen una mayor proporción de afiliados de mayor riesgo, intenta aumentar la competencia dentro del sistema y reducir las barreras de entrada a nuevas isapres.

Por otra parte, el plan ha generado discusiones en cuanto a la consistencia de sus beneficios. Por un lado, la instauración de la tarifa plana significará el aumento de la prima para determinados grupos de afiliados, que no podrán financiar el plan solamente con la cotización obligatoria. Según estimaciones propias, usando datos de 2010, al menos un 13\% de los afiliados, es decir, cerca

\footnotetext{
1 En este caso también se deja libre la opción de contratar un seguro complementario adicional.

2 Véase "Informe Comisión - Octubre 2011. Evaluar la factibilidad de crear un Plan Garantizado de Salud (PGS) al interior del sub-sistema de ISAPRE" [en línea] https://www.politopedia.cl/wp-content/uploads/2016/06/Informe-Comisi\%C3\%B3nPGS.2011.pdf.
} 
de 190.000 cotizantes, pagan una prima menor que la prima del PGS ${ }^{3}$. Por otro lado, el PGS no contempla la eliminación de las carencias y preexistencias a los usuarios que deseen trasladarse del sistema público a una isapre. La demanda del seguro público o privado de salud, por lo tanto, puede cambiar de acuerdo con los efectos que la nueva política producirá en los distintos grupos sociales. Algunos grupos se verán más atraídos por el sistema de isapres luego de la reforma, mientras que otros decidirán trasladarse al FONASA. Es natural esperar que las personas jóvenes cuyas primas se vean aumentadas y cuyo estado de salud represente bajos riesgos y por ende bajos costos de salud decidan trasladarse al FONASA 4 . Al mismo tiempo, las familias numerosas que tengan una elevada prima familiar, debido al pago per cápita de la tarifa del PGS, también son propicias a dejar el sistema privado de salud ${ }^{5}$. Así, una de las principales interrogantes se refiere a cómo la reforma impactará la demanda entre sistemas: isapres y FONASA. Este es precisamente el objetivo principal de la presente investigación. Sin embargo, cabe destacar la existencia de consideraciones y presiones sobre la oferta que escapan el ámbito de este estudio.

A fin de evaluar el efecto de la introducción del PGS en el sistema de isapres y el FONASA, es preciso desarrollar un modelo que permita determinar la demanda de las isapres y el FONASA para cada afiliado, considerando las características sociodemográficas, las características de la oferta de servicios y las preferencias de los usuarios ${ }^{6}$. Cabe tener en cuenta las siguientes variables: el nivel de ingresos, el riesgo, el tamaño del núcleo familiar, la edad, el género y las condiciones de la oferta de servicios de cada sistema en cada región del país.

Este modelo, por lo tanto, debe contemplar las características propias del sistema de salud chileno, como, por ejemplo, el hecho de que los trabajadores dependientes son obligados a contratar un seguro de salud con la cotización de un porcentaje de su salario y que, además, entre las alternativas de seguros, están los planes privados ofrecidos por las isapres y un plan único del sistema público, el FONASA, ambos con distintos esquemas de precio.

Este artículo se estructura de la siguiente forma. En la sección II se comenta la literatura relevante, tanto internacional como nacional. En la sección III se presenta el modelo de elección discreta, que introduce una serie de mejoras con respecto a los estudios previos en Chile. En la sección IV se realiza una descripción de la información utilizada para calibrar los modelos y la estadística descriptiva de las principales variables utilizadas. En la sección $V$ se muestran los resultados más importantes del estudio, en particular el impacto de la implementación del PSG sobre la demanda. Finalmente, a partir de los resultados, se presentan las principales conclusiones y recomendaciones de política del estudio.

\section{Revisión de la literatura}

A partir del trabajo seminal de Akerlof (1970), se ha prestado gran atención al efecto de la asimetría de información en la toma de decisiones en el mercado de la salud. Por un lado, los mercados de los seguros de salud son susceptibles de sufrir selección adversa debido a que los asegurados conocen

3 Según estimaciones de precio del PGS de Claudia M. Copetta, se requeriría una renta mínima de cerca de 320.000 pesos chilenos para que una sola persona se asegure con el PSG a través de la cotización obligatoria (Copetta, 2011). Cerca de 50.000 personas no podrían pagar el PGS con la cotización obligatoria; sin embargo, su estimación está sesgada debido a que no considera el efecto de las cargas en el precio final ni a los beneficiarios que pagan más que la cotización adicional (un $70 \%$ de la cartera de las isapres), muchos de los cuales también sufrirían alzas en los planes.

4 Según estimaciones propias, cerca de un 22\% de jóvenes menores de 25 años de edad pagan una prima menor que la que pagarían en el PGS, comparado con solo un 11\% del resto de la población.

5 Según estimaciones propias, cerca de un $25 \%$ de las familias con más de tres integrantes pagan una prima menor que la que pagarían en el PGS, comparado con solo un $8 \%$ de las familias con menos de tres integrantes.

6 El trabajo no modela ni estima la oferta, dada su gran complejidad. Por lo tanto, los resultados son solo equilibrios parciales. 
mucho más de sus propios riesgos de salud que los aseguradores, lo que lleva a que las coberturas muy generosas atraigan a riesgos altos. Por otra parte, la asimetría de información en el contexto de la relación médico-paciente puede contribuir a una sobreutilización de los servicios de salud, a medida que la cobertura de riesgos es mayor, caracterizando el riesgo moral.

En general, los estudios sobre la influencia del riesgo moral en las decisiones de elección de seguro de salud son derivados a partir de la teoría de maximización de la utilidad esperada. Friedman (1974), por ejemplo, sugiere un modelo de elección discreta para la elección entre seguros de salud, que permite realizar inferencias sobre la aversión al riesgo percibido respecto de las alternativas. El estudio, realizado para los Estados Unidos con objetivos exploratorios, concluye que los grupos familiares muestran menor aversión al riesgo en comparación con los individuos. En investigaciones posteriores, Feldman propone un análisis de la sensibilidad de la elección de los trabajadores estadounidenses con relación a las primas pagadas directamente por ellos para los planes de salud (Feldman y otros, 1989). Los resultados demuestran una alta sensibilidad de los individuos respecto a la prima pagada directamente de sus bolsillos. Cutler y Reber (1998) analizan los costos de la selección adversa usando información sobre cómo eligen sus planes de salud los empleados de la Universidad de Harvard. Los autores analizan una reforma realizada por la institución, que en 1995 cambió de un sistema de subsidios generosos a un sistema que solo subsidia una cantidad fija de cada seguro, independientemente del plan y la prima. Los resultados muestran que la reforma logró disminuir los costos asumidos por la Universidad, ya que los empleados empezaban a decidirse por planes más económicos.

Para el caso particular de Chile, existen pocas investigaciones en el ámbito del seguro de salud, que comenzaron a realizarse a partir de la década de 1990. Así, Sapelli y Torche (1998) analizan los determinantes en la elección de seguros de salud usando la Encuesta de Caracterización Socioeconómica Nacional (CASEN) de 1990 y 1994. Los autores concluyen que las variables más importantes para la decisión entre seguro público y privado son el ingreso, la edad y el sector de residencia del individuo. Además, constatan que la información privada respecto al estado de salud del individuo genera selección adversa en contra de los aseguradores privados, a la vez que las informaciones públicas respecto al estado de salud producen selección adversa en contra del asegurador público. En estudios posteriores, esta vez utilizando la CASEN de 1990 y 1996, Sapelli y Torche (2001) corroboran la importancia del ingreso y el sector de residencia en la determinación de la preferencia del individuo, pero también agregan la relevancia del precio del seguro y el gasto esperado en salud en términos de copagos. Los autores resaltan que, a un mayor gasto esperado en salud, el seguro público se torna más atractivo, razón por la cual el ingreso no puede ser la única variable explicativa para la elección entre seguros de salud. El estudio, sin embargo, presenta la limitante de usar una tabla de factores de riesgos de una única aseguradora privada. A pesar de que las tablas de factores de las aseguradoras privadas estén correlacionadas entre sí, las variaciones en la tarificación pueden deberse no solo al ajuste por riesgo, sino también a las distintas primas básicas cobradas en cada plan, las que dependen del tipo de cobertura ofrecido. Por lo anterior, el uso de una única tabla falla en representar el precio promedio de todas las aseguradoras. Para evitar este problema, en el presente trabajo se utiliza un vector de precios promedio que los cotizantes pagan de acuerdo con la edad, el género, el nivel de ingreso y el número de dependientes en la familia, lo que ya lleva incorporados los factores de riesgo, la prima básica y las preferencias de los individuos de hacer o no cotización adicional a la cotización obligatoria.

Por otro lado, Sapelli y Vial (2003) analizan la selección adversa y los gastos esperados en los sistemas de salud. El estudio ratifica el problema de selección adversa en el FONASA debido a variables observables en los modelos de decisión. Sin embargo, los resultados no acusan selección adversa en isapres, lo que se debería al diseño y variedad de estos planes, en que los precios discriminan por riesgo. 
Sanhueza y Ruiz-Tagle (2002) destacan la importancia de la composición del núcleo familiar respecto del género y la edad. No obstante, una de las mayores deficiencias en este estudio consiste en que los resultados de la variable de estado de salud indican que existe evidencia de selección adversa en contra del sistema privado, lo que va en contra de lo que se podría suponer en este particular sistema debido a las condiciones preexistentes.

En otro estudio, Tokman, Marshall y Espinosa (2007) concluyen que la probabilidad de que una persona esté afiliada a una isapre aumenta con el ingreso del cotizante y disminuye con el riesgo de los integrantes del grupo familiar. Además, agregan que la probabilidad de afiliarse a una isapre aumenta en función de la disponibilidad de camas privadas en la región en que habita, entre otros factores.

Por otro lado, Dawes Ibáñez (2010) analiza los efectos de la implementación del plan GES en la elección entre sistema de salud público y privado. Para esto, comparan las preferencias de los afiliados por los dos sistemas de salud antes y después de la implementación de la reforma, utilizando los datos de las encuestas CASEN de 2000 y 2006. Los autores utilizan el enfoque de Sapelli y Torche (2001), incorporando los aportes de Tokman, Marshall y Espinosa (2007). Los resultados del modelo antes de la reforma concuerdan con los estudios anteriores, destacando la importancia del ingreso y el riesgo en la selección del sistema de salud y agregando además la relación positiva de tener un cónyuge, del nivel de educación y de trabajar en grandes firmas con la probabilidad de optar por el seguro de salud privado. En el análisis posterior a la reforma, los resultados apuntan a una menor influencia del riesgo de salud individual en la preferencia del individuo, lo que según los autores obedecería a la mejor cobertura de riesgos catastróficos implementados por el plan GES, creando un conjunto de riesgos que reduce la diferencia de precios pagados a los seguros privados. Sin embargo, una limitante importante en el estudio de Dawes Ibáñez (2010) es haber comparado las situaciones anterior y posterior a la reforma con una ventana de tiempo de seis años entre ellas, un intervalo considerablemente grande, en que muchos otros factores pueden haber influenciado los cambios en las preferencias por el seguro de salud ${ }^{7}$.

Finalmente, la investigación llevada a cabo por Duarte (2012) analiza la elasticidad precio de los gastos de salud, indicando posibles preferencias que pueden ser utilizadas en un análisis de elección de planes. Duarte utiliza datos compilados de registros del mercado privado de seguros de salud chileno y muestra que los individuos de altos ingresos son cinco veces más sensibles al precio que los individuos de bajo nivel de ingresos. El estudio, sin embargo, no abarca el sistema público, pues trabaja solamente con información de afiliados a isapres.

De las investigaciones antes señaladas podemos inferir que para el caso chileno se han concentrado en medir el efecto de los precios y del riesgo en la elección de los individuos, pero poco se ha hecho para analizar la influencia de la calidad de los sistemas y su impacto en la elección del seguro de salud. El presente estudio refina este análisis, agregando el componente de calidad para mejorar la capacidad explicativa del modelo. Se espera, con esto, poder identificar de mejor manera la preferencia de los individuos y los patrones de sustitución entre los distintos sistemas de salud. Lo anterior permite desarrollar una predicción más precisa del impacto del PGS y obtener mejores resultados en la caracterización de los grupos afectados por la reforma.

\footnotetext{
7 Durante estos años, por ejemplo, hubo una importante disminución de los indicadores de desigualdad en Chile, lo que puede influenciar en la disminución del efecto del ingreso. Además, el número de planes en isapres disminuyó de 16.696 en diciembre de 2003 a 7.454 en enero de 2006, lo que indica una menor discriminación de precios de los planes privados como resultado de las restricciones implementadas por la Ley de Isapres. Como la discriminación de precios se caracteriza por la creación de planes más asequibles a personas de menores niveles de ingreso, lo anterior podría ser un factor relevante para que el sistema privado de salud pase a ser menos atractivo. En esta misma línea, otra limitante consiste en el hecho de que no se puede analizar la implementación de las GES utilizando grupos de control, ya que se aplica tanto a cotizantes en isapres como en el FONASA. Como las GES son un nuevo atributo tanto del seguro público como del seguro privado, no se puede determinar, por lo tanto, que la preferencia por uno u otro sea resultante de su implementación.
} 


\section{El modelo}

El modelo de decisión desarrollado en este estudio corresponde a un modelo de elección discreta, que simula la demanda de planes de salud privados y no toma en consideración cambios estructurales a la oferta. Para que un modelo se ajuste al marco de elección discreta, las alternativas disponibles para elegir deben ser mutuamente excluyentes, el grupo de alternativas debe contener todas las opciones disponibles para el individuo que toma la decisión y debe tener un número finito de opciones. El individuo elige, entre todas las alternativas disponibles, aquella que le entregue mayor utilidad.

Para el modelamiento de la función de utilidad del individuo, se utiliza el enfoque de utilidad aleatoria, cuya aplicación a la economía fue propuesta inicialmente por McFadden y luego desarrollada por Manski. De acuerdo con esto, las funciones de utilidad son tratadas como aleatorias, no para reflejar la falta de racionalidad, sino la falta de información con relación a las características de las alternativas o de los individuos por parte del observador. Este modelo supone que la utilidad del individuo $\left(U_{i}\right)$ se puede separar aditivamente en un componente determinístico observable $\left(\varepsilon_{i}\right)$ y un componente aleatorio no observable $\left(V_{i}\right)$ para cada alternativa. Así, considerando individuos que eligen distintos sistemas de seguro, tenemos:

$$
\begin{gathered}
U_{i I}=V_{i I}+\varepsilon_{i I} \\
U_{i F}=V_{i F}+\varepsilon_{i F}
\end{gathered}
$$

Donde $U_{i I}$ corresponde a la utilidad que el individuo $i$ obtiene del seguro de salud isapre (subíndice I), mientras que $U_{i F}$ es la utilidad que el individuo $i$ recibe si elige el seguro del FONASA (subíndice F). El componente $\varepsilon_{i}$ captura todos los factores que afectan la utilidad, pero no están incluidos en $V_{i}$. La probabilidad de que el individuo $i$ seleccione el sistema privado de salud es, entonces:

$$
\begin{gathered}
P_{i f}=\operatorname{Prob}\left(U_{i I}>U_{i F}\right) \\
=\operatorname{Prob}\left(V_{i I}+\varepsilon_{i I}>V_{i F}+\varepsilon_{i F}\right) \\
=\operatorname{Prob}\left(\varepsilon_{i F}+\varepsilon_{i I}<V_{i I}+V_{i F}\right)
\end{gathered}
$$

Esto consiste en la probabilidad acumulada de que cada término aleatorio $\varepsilon_{i F-} \varepsilon_{i I}$ se encuentre por debajo del valor observado para $V_{i I-} V_{i F}$. Por ejemplo, para modelos logit, la distribución del error se distribuye como la función de densidad valor extremo. Por lo tanto, obtenemos:

$$
P_{i I}=\int I\left(\varepsilon_{i F}-\varepsilon_{i I}<V_{i I}-V_{I F}\right) f\left(\varepsilon_{i}\right) d \varepsilon_{i}
$$

donde $I(\cdot)$ es la función indicador, igual a 1 cuando la expresión entre paréntesis es verdadera y 0 en caso contrario. En este marco teórico, asumimos las siguientes funciones de utilidad:

$$
\begin{gathered}
U_{i I}=\alpha\left(Y_{i}-P_{i I}\right)+\beta X_{i I}+\gamma X_{i I} Z_{i}+\delta Z_{i}+\varepsilon_{i I} \\
U_{i F}=\alpha\left(Y_{i}-P_{i F}\right)+\beta X_{i F}+\gamma X_{i F} Z_{i}+\delta Z_{i}+\varepsilon_{i F}
\end{gathered}
$$

Donde

$Y_{i}=$ Ingreso del individuo;

$P_{i I}=$ Precio del seguro privado cobrado por la isapre para el individuo $i$;

$P_{i F}=$ Precio del seguro público del FONASA para el individuo $i$, correspondiente al $7 \%$ del ingreso individual, o 0,07 $Y_{i}$; 
$X_{i I}=$ Vector de atributos que el seguro de salud isapre ofrece al individuo $i$;

$X_{i F}=$ Vector de atributos que el seguro de salud del FONASA ofrece al individuo $i$;

$Z_{i}=$ Vector de características sociodemográficas del individuo $i$;

Las ecuaciones (5) y (6) representan la función de utilidad de las isapres y el FONASA para el individuo $i$, respectivamente. Se asume que las preferencias son estables a través del tiempo.

Se asume que la utilidad esperada para cada alternativa depende positivamente del ingreso neto luego de haberse pagado la prima del seguro. Por lo tanto, se espera que, a medida que el seguro de salud se torna más caro, su utilidad esperada disminuye. La utilidad esperada también depende de los atributos del servicio entregado en el plan: a una mayor calidad en la entrega de estos servicios, mayor será la utilidad esperada derivada de este. Esto está motivado por un estudio de opinión de la Superintendencia de Salud de Chile, que muestra que entre las características más valoradas por los usuarios del sistema privado está la calidad de la atención médica, la rapidez de la atención y la calidad de los médicos, a la vez que en el sistema público una de las características más valoradas es el bajo costo de la atención, es decir, bajos niveles de copago (Superintendencia de Salud, 2012). Las características sociodemográficas del individuo, por otra parte, también influyen en el nivel de utilidad percibido por un seguro de salud. Una persona más adversa al riesgo, por ejemplo, tiende a valorar una mayor cobertura y ser menos sensible al precio, mientras que un individuo que reside en zonas rurales donde existan pocos hospitales del sistema privado tiende a valorar más el seguro público de salud.

Al decidir qué sistema de salud elegir, cada individuo evalúa la diferencia entre la utilidad de estar en el sistema de isapres y la utilidad de estar en el FONASA. Por lo tanto, elige la opción que maximiza su utilidad. De esta forma, si la diferencia es positiva, se elige el seguro privado; si la diferencia es negativa, se elige el seguro público.

Restando la ecuación (5) en (6), se tiene:

$$
U_{i I}-U_{i F}=\alpha\left(0.07 Y_{i}-P_{i I}\right)+\beta \Delta X_{i}+\gamma \Delta X_{i} Z_{i}+\Delta \varepsilon_{i}
$$

A diferencia de lo desarrollado anteriormente, el modelo aquí propuesto define que el vector de atributos del seguro de salud se compone de: i) un vector de características de calidad y servicio observables, el $\Delta X_{i 1}$; y ii) un vector de características no observables $\Delta X_{i 2}$. Finalmente, se tiene que $\Delta X_{i=} \Delta X_{i 1}+\Delta X_{i 2}$, por lo que:

$$
U_{i I}-U_{i F}=\alpha\left(0.07 Y_{i}-P_{i I}\right)+\beta\left(\Delta X_{i 1}+\Delta X_{i 2}\right)+\gamma\left(\Delta X_{i 1}+\Delta X_{i 2}\right) Z_{i}+\Delta \varepsilon_{i}
$$

En la estimación, se asume un no observable para cada opción (FONASA e isapre), por lo tanto, la diferencia para este no observable es normalizada a 1. Como las características sociodemográficas del individuo son las mismas independientemente de su elección, se tiene finalmente que:

$$
I=U_{i I}-U_{i F}=\alpha\left(0.07 Y_{i}-P_{i I}\right)+\beta_{1} \Delta X_{i 1}+\beta_{2} \Delta X_{i 2}+\gamma_{1} \Delta X_{i 1} Z_{i}+\gamma_{2} Z_{i}+\Delta \varepsilon_{i}
$$

A diferencia de estudios anteriores, el modelo deja de usar el factor de riesgo individual como variable sustitutiva del precio del seguro privado y utiliza un vector de precios estimados de acuerdo con la edad, el número de cargas y el nivel de ingreso del cotizante ${ }^{8}$. El uso del vector de precio en

8 Estimación propia a partir de datos extraídos de Duarte (2012). 
lugar de la variable sustitutiva de riesgo individual permite que el nuevo precio estimado para el Plan Garantizado de Salud sea usado como contrafactual en el modelo de decisión, lo que permite simular la elección de los individuos entre seguro público y privado luego de la implementación de la reforma. En particular, el estudio aborda la evaluación de una prima comunitaria única. Ahora bien, este precio promedio, si bien no es el precio que el individuo en promedio observa, y por lo tanto sesga los resultados, es una mejor aproximación que la usada previamente en la literatura.

Una vez conocido cuál será el efecto de la reforma en el tamaño relativo de ambos sistemas y qué características, en términos de ingresos, edad, tamaño familiar y estado de salud poseen los individuos que se cambian de un sistema a otro, este análisis permitirá estimar el costo fiscal eventual de la reforma, en la medida en que se trasladen al FONASA afiliados que gastan más que lo que contribuyen.

Dado que solo la demanda está modelada en esta investigación, implícitamente se asume un escenario donde la oferta se ajusta de modo de mantener la renta económica que percibe el sector constante. Para mantener la renta económica se requiere un aumento significativo del precio del PGS, lo cual explica la caída de la demanda del servicio de las isapres. Este puede ser considerado entonces un escenario extremo. Si las isapres cuentan con grandes rentas económicas, podrían por ejemplo disminuir el impacto de la reforma, absorbiendo los mayores costos que requiere este sistema más solidario, generando un incremento de precios menor, disminuyendo el precio final a sus afiliados y reduciendo así la caída de su demanda.

\section{Datos}

Los datos utilizados en este estudio provienen de la Encuesta de Caracterización Socioeconómica Nacional 2013, que entrega información respecto a las condiciones de vivienda, situación laboral, nivel educacional, condiciones de salud y características demográficas de los individuos. La encuesta, aplicada entre noviembre de 2013 y enero de 2014 a 66.724 hogares del país, cuenta con representatividad nacional, por zona urbana y rural, para las 15 regiones del país y para un total de 324 comunas.

Uno de los requisitos necesarios para utilizar el modelo de elección discreta consiste en que las alternativas presentadas a un individuo deben ser mutuamente excluyentes. Por esta razón el modelo de decisión se aplica solamente a empleados, obreros o jubilados, debido a que estos individuos están sujetos a la cotización obligatoria de salud y, por lo tanto, deben elegir obligatoriamente entre contratar un plan en una isapre o afiliarse al FONASA, pagando en ambos casos al menos el 7\% de su salario.

El modelo desarrollado se aplica al jefe del núcleo familiar, debido a que es él quien elige a qué seguro de salud se afiliará, decisión determinada por sus características individuales y de su núcleo familiar y por los atributos asociados a las alternativas disponibles. La encuesta CASEN 2013 contiene 218.490 observaciones, distribuidas en 78.938 núcleos familiares. La muestra expandida cuenta con una población de 17.273.085 personas, distribuidas en 6.256.912 hogares.

Como se mencionó, la muestra para análisis incluye solamente a los empleados, obreros y jubilados con ingreso imponible superior a cero y que estén afiliados al sistema de isapres o al FONASA grupos B, C y D. En el cuadro 1 se presenta la distribución del total de afiliados entre los sistemas de salud en 2013. 
Cuadro 1

Chile: distribución de afiliados por quintil de ingreso y seguro de salud, 2013

(En porcentajes)

\begin{tabular}{lcccccc}
\hline & Quintil I & Quintil II & Quintil III & Quintil IV & Quintil V & Total \\
\hline FONASA & 11,0 & 18,4 & 17,6 & 15,6 & 7,0 & 69,6 \\
\hline Isapres & 0,7 & 0,5 & 1,6 & 4,6 & 15,2 & 22,6 \\
\hline Otros & 1,2 & 1,2 & 1,2 & 2,1 & 2,0 & 7,7 \\
\hline Total & 12,9 & 20,1 & 20,4 & 22,4 & 24,2 & 100,0 \\
\hline
\end{tabular}

Fuente: Elaboración propia, sobre la base de datos de la Encuesta de Caracterización Socioeconómica Nacional (CASEN) 2013.

En el cuadro 2 se presenta la proporción del total de afiliados entre el sistema público y privado en 2013 de la muestra final. En el cuadro 3 se detallan algunas características demográficas de la muestra final.

\section{Cuadro 2}

Chile: distribución de afiliados por quintil de ingreso y seguro de salud después de limpieza de la muestra, 2013

(En porcentajes)

\begin{tabular}{lcccccc} 
& Quintil I & Quintil II & Quintil II & Quintil IV & Quintil V & Total \\
\hline FONASA & 9 & 21 & 20 & 17 & 7 & 74 \\
\hline Isapres & 1 & 1 & 2 & 5 & 17 & 26 \\
\hline Total & 9,3 & 21,8 & 21,9 & 22,7 & 24,2 & 100,0 \\
\hline
\end{tabular}

Fuente: Elaboración propia, sobre la base de datos de la Encuesta de Caracterización Socioeconómica Nacional (CASEN) 2013.

Una importante desventaja en el uso de encuestas es que se cuenta con información declarada por los propios cotizantes, que puede estar sujeta a serias imprecisiones. En este caso, por ejemplo, la información de los ingresos individuales corresponde al valor declarado como ingreso neto, y no ingreso bruto. Para el uso en el modelo, este valor es transformado a ingreso imponible, reajustándose los valores correspondientes del impuesto a la renta y las cotizaciones de salud y de fondos de pensiones. Además, la CASEN 2013 no contiene la información efectiva del número de cargas por afiliado, por lo que se consideran como cargas aquellos miembros del núcleo familiar menores de 18 años, o bien mayores de 18 años y menores de 25 que asistan a algún establecimiento educacional. Adicionalmente, no se puede distinguir cómo se distribuyen las cargas en el caso en que ambos cónyuges sean activos y coticen para el sistema de salud. Por esta razón, se supone que el jefe del núcleo familiar es quien toma la decisión y asume todas las cargas.

La prima del seguro público, es decir el $7 \%$ del salario, se estimó utilizando una variable que representa el salario del jefe del núcleo familiar. Si el cónyuge es empleado, obrero o jubilado, se atribuye su sueldo a esta variable, lo que define un "cotizante único", que se hace cargo de todas las cargas familiares. Esta simplificación disminuye el problema de la falta de información sobre la asignación de las cargas entre los cónyuges. Sin embargo, obliga a asumir que los cónyuges que son activos y cotizan eligen el mismo sistema de salud.

Para la prima del seguro privado, como se mencionó anteriormente, se utilizará una estimación promedio por edad, número de cargas y nivel de ingreso del cotizante, usando datos de la Superintendencia de Isapres.

Las otras variables que se utilizan en este estudio corresponden a características individuales de los encuestados, empleadas con frecuencia en los estudios revisados para el caso chileno. Estas variables sirven para ser usadas por sí solas en el modelo, así como para interactuar con las variables que contienen los atributos relacionados con la calidad y el servicio de los seguros de salud público y privado. Entre ellas, se mencionan: edad, un indicador del género, un indicador de si el jefe del núcleo 
posee cónyuge, número de cargas y dos indicadores de ubicación geográfica (uno correspondiente a Santiago y el otro a si el individuo vive en una ciudad pequeña). En los cuadros 3 y 4 se muestran algunas estadísticas de estas variables.

\section{Cuadro 3}

Chile: estadísticas descriptivas, por quintil y sistema, 2013

(En porcentajes, años cumplidos, pesos chilenos y número)

\begin{tabular}{llccccc}
\hline & Sistema & Quintil I & Quintil II & Quintil III & Quintil IV & Quintil V \\
\hline \multirow{2}{*}{ Cotizantes } & FONASA & 94,3 & 97,3 & 91,0 & 76,5 & 28,7 \\
\cline { 2 - 7 } Cotizantes mujeres & Isapres & 5,7 & 2,7 & 9,0 & 23,5 & 71,4 \\
\hline \multirow{2}{*}{ Cotizantes hombres } & FONASA & 97,3 & 97,7 & 95,6 & 79,7 & 33,6 \\
\cline { 2 - 7 } & Isapres & 2,7 & 2,3 & 4,4 & 20,3 & 66,4 \\
\hline \multirow{2}{*}{ Edad promedio (años cumplidos) } & FONASA & 92,3 & 97,0 & 89,5 & 75,4 & 27,3 \\
\cline { 2 - 7 } & Isapres & 7,7 & 3,0 & 10,5 & 24,6 & 72,7 \\
\hline \multirow{2}{*}{$\begin{array}{l}\text { Sueldo imponible promedio } \\
\text { (pesos chilenos) }\end{array}$} & FONASA & 51,3 & 49,1 & 46,7 & 46,7 & 47,1 \\
\hline \multirow{2}{*}{ Riesgo promedio } & FONASA & 198771 & 271453 & 364389 & 549420 & 1181443 \\
\cline { 2 - 7 } & Isapres & 207345 & 290311 & 415292 & 646902 & 2232254 \\
\hline \multirow{2}{*}{\begin{tabular}{l} 
Dependientes \\
\cline { 2 - 7 }
\end{tabular}} & FONASA & 3,5 & 3,4 & 3,2 & 3,4 & 3,5 \\
\hline & Isapres & 3,6 & 3,0 & 2,6 & 2,8 & 2,8 \\
\cline { 2 - 6 } & FONASA & 2,1 & 2,3 & 2,4 & 2,4 & 2,6 \\
\hline
\end{tabular}

Fuente: Elaboración propia, sobre la base de datos de la Encuesta de Caracterización Socioeconómica Nacional (CASEN) 2013.

\section{Cuadro 4}

Chile: estadísticas descriptivas de variables independientes sociodemográficas, 2013

\begin{tabular}{lcc}
\hline Variable & Promedio & Desviación estándar \\
\hline Edad & 43,25 & 12,36 \\
\hline Educación & 5,88 & 3,25 \\
\hline Cónyuge & 0,25 & 0,43 \\
\hline Santiago o Región Metropolitana & 0,51 & 0,50 \\
\hline Ciudades pequeñas & 0,41 & 0,49 \\
\hline Estado de salud & 5,75 & 1,14 \\
\hline Aversión al riesgo & 0,16 & 0,37 \\
\hline Preexistencia & 0,23 & 0,42 \\
\hline Días de hospitalización & 0,01 & 0,11 \\
\hline Riesgo & 3,08 & 1,30 \\
\hline Número de personas & 2,36 & 1,16 \\
\hline
\end{tabular}

Fuente: Elaboración propia, sobre la base de datos de la Encuesta de Caracterización Socioeconómica Nacional (CASEN) 2013.

También se utilizan variables relacionadas al estado de salud, como: dos indicadores del estado de salud (autorreportado) de los individuos. El primero toma el valor 1 si el individuo se autoevalúa con notas 1 o 2, es decir, "muy mal" o "mal". El segundo indicador toma el valor 1 si el encuestado se autoevalúa 3 o 4, es decir, "menos que razonable" o "razonable". Por lo tanto, quedan las notas 5, 6 y 7, que corresponden a "más que razonable", "bueno" y "muy bueno". Estas variables intentan capturar tendencias en la selección adversa y riesgo moral después de la implementación de la reforma. Se construye una variable que contiene la suma de los factores de riesgo de las cargas y el jefe del núcleo familiar. Esta variable se basa en una tabla de factor de riesgo actualizada a 2009, calculada a partir de un promedio ponderado entre las tablas de isapres abiertas en 2005 y la proporción de beneficiarios de cada isapre abierta de 2009. 
Finalmente, el modelo incorpora variables de calidad y servicio que son necesarias para representar en otra dimensión los atributos de los seguros de salud. Primero, se construye, usando datos del Departamento de Estadísticas e Información de Salud (DEIS) del Ministerio de Salud ${ }^{9}$, el número de establecimientos de salud disponibles por cada 100.000 habitantes en cada región por cada sistema, como se observa en el cuadro 5 . Segundo, se construye una variable promedio del gasto de los cotizantes en ambos sistemas, por región ${ }^{10}$.

Cuadro 5

Chile: número de establecimientos de salud, por región, 2013 (Por 100.000 habitantes)

\begin{tabular}{lcc}
\hline Región & Isapres & FONASA \\
\hline$I$ & 0,65 & 0,33 \\
\hline$I$ & 1,58 & 0,35 \\
\hline$I I$ & 1,08 & 0,36 \\
\hline$I V$ & 0,28 & 0,42 \\
\hline$V$ & 0,63 & 0,46 \\
\hline$V I$ & 0,34 & 0,23 \\
\hline$V I I$ & 0,30 & 0,30 \\
\hline$V I I I$ & 0,59 & 0,40 \\
\hline$I X$ & 0,31 & 0,31 \\
\hline$X$ & 0,24 & 0,36 \\
\hline$X I$ & 0,96 & 0,96 \\
\hline$X I I$ & 1,27 & 0,63 \\
\hline RM & 1,03 & 0,34 \\
\hline$X I V$ & 0,53 & 0,26 \\
\hline$X V$ & 3,23 & 0,54 \\
\hline
\end{tabular}

Fuente: Elaboración propia, sobre la base de datos del Departamento de Estadísticas e Información de Salud del Ministerio de Salud.

En el cuadro 6 se presentan las diferencias de los promedios de las variables de calidad y servicio, por quintil de ingreso.

\section{Cuadro 6}

Chile: variables de calidad, por quintil de ingreso, 2009

(En pesos chilenos)

\begin{tabular}{lccccc}
\hline & Quintil I & Quintil II & Quintil III & Quintil IV & Quintil V \\
\hline Promedio diferencia copago & 20178 & 17502 & 14862 & 12809 & 9254 \\
\hline Promedio diferencia hospitales por habitante & 0,32 & 0,43 & 0,53 & 0,56 & 0,64 \\
\hline
\end{tabular}

Fuente: Elaboración propia, sobre la base de datos de la Encuesta de Protección Social (EPS) de 2009.

9 Se utiliza la base de datos de egresos hospitalarios más recientes del DEIS para 2011. Para construir el índice, se seleccionan solamente aquellos catalogados como establecimientos de mayor complejidad. No se consideran postas de salud rural, centros de salud urbanos, familiares, comunitarios familiares o rurales; vacunatorios, establecimientos de mediana o menor complejidad, hospitales de campaña u hospitales militares de campaña, Servicio de Atención Primaria de Urgencia (SAPU); Clínica Dental Móvil, oficina sanitaria, clínica en convenio, Consultorio General Rural, centros de atención cerrada; Centros de Referencia de Salud, Centros de Diagnóstico Terapéutico y hospitales delegados. Para los establecimientos privados, se mantienen solamente hospitales y clínicas.

10 El promedio se calcula incluyendo consultas médicas de urgencia y especialidad, rayos x o ecografías y controles preventivos. El promedio no incluye gastos por hospitalización o cirugías. Los promedios se obtienen a partir de datos de la Encuesta de Protección Social (EPS) de 2009; se optó por no trabajar con la última EPS disponible de 2012, ya que se considera un producto no logrado por parte de la Subsecretaría de Previsión Social. 
Por último, se utilizan otras variables con el objetivo de replicar resultados anteriores, tales como: el logaritmo del ingreso del jefe del núcleo, una variable de precio (factor de riesgo) en dos versiones, una con los mismos datos usados por Sapelli y Torche (2001) y otra actualizada al año 2009, calculada a partir de un promedio ponderado entre las tablas de isapres abiertas en 2005 y la proporción de beneficiarios de cada isapre abierta de 2009, y el número de personas en el núcleo familiar que han sido hospitalizadas por más de 8 días en los tres meses anteriores a la encuesta.

\section{Resultados}

La demanda del seguro de salud privado es una función de la diferencia de precios entre el seguro público y el privado: mientras más negativa esta diferencia, mayor la probabilidad de que el individuo se afilie a una isapre. La ecuación también incluye atributos de calidad y servicio de los seguros, descritos en el vector de atributos observables: cuanto mejor sea la calidad y el servicio de los planes de las isapres en comparación con el FONASA, mayor la probabilidad de que el individuo elija una isapre. Debido a la normalización del vector de atributos no observables, las características sociodemográficas del individuo representan por sí solas un efecto en la preferencia por un plan de salud. Asimismo, las características individuales, como la edad, el ingreso, el sexo, el sector de residencia, entre otras, influyen en cómo las personas perciben los atributos específicos de cada plan. Por esta razón, se incluyen una serie de interacciones entre variables sociodemográficas del individuo y variables de calidad y servicio de los seguros de salud, con el objetivo de caracterizar de mejor manera las preferencias heterogéneas de la población. El principal interés del análisis está en estudiar la sensibilidad del individuo frente a cambios en la prima del seguro de salud, considerando las variables sociodemográficas y las variables de calidad. Este enfoque permite analizar cómo la reforma puede incidir en el fenómeno de selección adversa, efecto que podría afectar al seguro público debido a que este no posee restricciones para recibir a afiliados de cualquier tipo de riesgo o costo esperado para el sistema.

\section{Comparación de los resultados con la literatura}

La variable de interés es una variable binaria que toma el valor 1 si el individuo elige el sistema de isapres y 0 si elige el FONASA. En el cuadro 7 se muestran los resultados de la regresión para el modelo propuesto por Sapelli y Torche. La regresión A replica el modelo usando la misma tabla de factor de riesgo usada en el modelo original; la B utiliza la tabla de factor de riesgo actualizada y, finalmente, la $\mathrm{C}$ utiliza el vector de precios construidos sobre la base de los precios reales que pagan los individuos.

Al observar la regresión, se advierte que la sensibilidad al precio ha dejado de ser significativa para 2013 (regresión A), al tiempo que la relevancia de la sensibilidad al ingreso se ha mantenido. Además, el uso del factor de riesgo como variable sustitutiva de la prima privada (regresión B) se muestra no significativo, a diferencia del precio creado a partir de las primas reales (regresión C). De lo anterior podemos inferir que el factor de riesgo no sería la variable sustitutiva más eficiente del precio promedio. Esto se debe a que el factor de riesgo no incorpora el efecto de la prima básica, que tiene un valor diferenciado para cada plan. 
Cuadro 7

Chile: coeficientes y desviaciones estándar de las regresiones

\begin{tabular}{|c|c|c|c|c|c|c|c|}
\hline \multirow{2}{*}{ Variables $^{\mathrm{a}}$} & $\begin{array}{c}\text { Sapelli y Torche } \\
\text { CASEN } 1996\end{array}$ & Regresión A & CASEN2013 & Regresión B & CASEN2013 & Regresión C & CASEN2013 \\
\hline & Coeficientes & Coeficientes & $\begin{array}{l}\text { Desviación } \\
\text { estándar }\end{array}$ & Coeficientes & $\begin{array}{l}\text { Desviación } \\
\text { estándar }\end{array}$ & Coeficientes & $\begin{array}{l}\text { Desviación } \\
\text { estándar }\end{array}$ \\
\hline L_ingreso & $2,10^{\star \star}$ & $2,647^{\text {***}}$ & 0,199 & $2,650^{\star \star \star}$ & 0,198 & $2,906^{* \star \star}$ & 0,183 \\
\hline Precio & $-0,20^{\text {** }}$ & $-0,105$ & 0,079 & $-0,081$ & 0,077 & $-0,0142^{\star \star}$ & 0,005 \\
\hline Cónyuge & $0,47^{\star \star}$ & $0,332^{\star \star \star}$ & 0,079 & $0,301^{\star \star \star}$ & 0,081 & $0,439^{\star \star \star}$ & 0,075 \\
\hline Edad & $-0,04^{\star *}$ & $-0,004$ & 0,003 & $-0,002$ & 0,003 & $-0,0101^{\star *}$ & 0,003 \\
\hline Días_Hosp & $-0,49$ & $-0,002$ & 0,102 & 0,002 & 0,094 & $-0,044$ & 0,095 \\
\hline Santiago_RM & $5,11^{\star *}$ & 3,824 & 2,905 & 4,140 & 2,898 & $5,875^{\star}$ & 2,750 \\
\hline Ciud_Pequeñas & $3,32^{\star *}$ & 4,724 & 2,912 & 4,961 & 2,908 & $7,048^{*}$ & 2,755 \\
\hline Lin_Stgo & $-0,29^{*}$ & $-0,168$ & 0,218 & $-0,174$ & 0,217 & $-0,348$ & 0,208 \\
\hline Lin_Pequeñas & $-0,18$ & $-0,290$ & 0,218 & $-0,301$ & 0,218 & $-0,481^{*}$ & 0,209 \\
\hline Pre_Stgo & $-0,05$ & $-0,127$ & 0,092 & $-0,170$ & 0,087 & 0,000 & 0,000 \\
\hline Pre_Pequeñas & $-0,03$ & $-0,054$ & 0,088 & $-0,065$ & 0,084 & 0,000 & 0,000 \\
\hline Constante & $-24,84^{\star \star *}$ & $-36,91^{\star * \star}$ & 2,644 & $-37,01^{\text {** }}$ & 2,63 & $-40,21^{\star \star *}$ & 2,407 \\
\hline Pseudo- $R^{2}$ & 0,26 & 0,377 & & 0,378 & & 0,373 & \\
\hline
\end{tabular}

Fuente: Elaboración propia.

Nota: $\quad{ }^{* * *}$ significativo al 1\%; ${ }^{* *}$ significativo al $5 \%$; *significativo al $10 \%$

a Estas variables están definidas en Sapelli y Torche (1998): L_ingreso (logaritmo del ingreso); Precio (el índice construido); Cónyuge (variable dummy); Edad (edad); Días_Hosp (número de familiares que han sido hospitalizados por más de ocho días en los últimos tres meses); Santiago_RM (variable dummy que identifica a Santiago (Chile)); Ciud__Pequeñas (variable dummy que identifica ciudades pequeñas); Lin_Stgo y Lin_Pequeñas (interacciones entre las variables dummy de ciudades y el logaritmo del ingreso); Pri_Stgo y Pri_Pequeñas (interacciones entre las variables dummy de ciudades y el precio).

\section{Resultados del modelo propuesto}

El cuadro 8 muestra los resultados de la regresión para el modelo propuesto. La variable Dif_Precio es la diferencia entre la prima pública menos la prima privada. Como se esperaba, mientras más negativa es la diferencia de precios entre seguro público y seguro privado, menor es la probabilidad de afiliación al sistema de isapres. Las otras variables relacionadas con atributos de los seguros de salud fueron construidas como el valor que toman en el seguro privado menos el valor que toman en el seguro público.

Una mayor diferencia en los valores de copagos entre las isapres y el FONASA implica una mayor probabilidad de afiliarse al FONASA. Por otro lado, una mayor diferencia entre el número de establecimientos en la región del individuo aumenta su probabilidad de afiliación al sistema de isapres.

El signo positivo encontrado para la variable Edad indica un aumento en el perfil de riesgo de los afiliados a isapres. Una posible razón para este efecto es la implementación de la Cobertura Adicional por Enfermedades Catastróficas (CAEC) en 2006, la que puede haber afectado a aquellas personas con perfil de riesgo de salud más alto, tornando el seguro de salud privado más atractivo para ellas. Aun así, la variable Estado_Salud, que captura informaciones no disponibles para las aseguradoras respecto al riesgo de salud del individuo, indica una preferencia por el seguro público en el caso de que existan evidencias de peor estado de salud.

Por otra parte, la existencia de un cónyuge activo y que cotice para algún sistema de salud aumenta la probabilidad de afiliación a isapres, debido a la interacción entre las variables Dif_Copago_sis y Cónyuge y a pesar del mayor valor en copagos que debe realizar en el sistema privado. Esto se debe a que la existencia de un cónyuge activo aumenta el ingreso familiar, lo que explica una mayor preferencia por el sistema de isapres.

En promedio, las variables Santiago_RM y Ciud_Pequeñas muestran que las personas que viven en esos lugares tienen mayores preferencias por isapres. Además, como se esperaba, el aumento de ingreso aumenta la probabilidad de afiliación al sistema de isapres. 


\section{Cuadro 8}

Chile: coeficientes y desviaciones estándar de la regresión del modelo propuesto

\begin{tabular}{|c|c|c|}
\hline & Coeficientes & Desviación estándar \\
\hline Dif_Precio & 0,076 & 0,047 \\
\hline Dif_Copago_Sis & $-0,647$ & 0,740 \\
\hline Dif_Hosp_Hab & 0,763 & 0,505 \\
\hline Ysueldo & $0,000^{* * *}$ & 2,61E-07 \\
\hline Edad & 0,002 & 0,006 \\
\hline Sexo & $-0,185$ & 0,119 \\
\hline Cónyuge & 0,323 & 0,177 \\
\hline Estado_Salud1 & 0,083 & 0,616 \\
\hline Estado_Salud2 & $-0,511$ & 0,272 \\
\hline Santiago_RM & $1,495^{\star \star \star}$ & 0,297 \\
\hline Ciud_Pequeñas & $0,797^{\star \star}$ & 0,265 \\
\hline Num_Pers & 0,003 & 0,0818 \\
\hline Dif_Cop x Ysueldo & 0,000 & 4,85E-07 \\
\hline Dif_Cop x Edad & $-0,000$ & 0,011 \\
\hline Dif_Cop x Sexo & 0,152 & 0,234 \\
\hline Dif_Cop x Cónyuge & 0,325 & 0,221 \\
\hline Dif_Cop x Salud1 & 0,739 & 0,613 \\
\hline Dif_Cop x Salud2 & 0,555 & 0,313 \\
\hline Dif_Cop x Numpers & $-0,069$ & 0,144 \\
\hline DifHosp x Ysueldo & 0,000 & $5,08 \mathrm{E}-07$ \\
\hline DifHosp x Edad & $-0,012^{*}$ & 0,005 \\
\hline DifHosp x Sexo & $-0,203$ & 0,145 \\
\hline DifHosp x Cónyuge & $-0,593^{\star \star \star}$ & 0,154 \\
\hline DifHosp x Salud1 & $-0,312$ & 0,386 \\
\hline DifHosp x Salud2 & 0,109 & 0,163 \\
\hline DifHosp x Santiago_RM & $0,889^{*}$ & 0,383 \\
\hline DifHosp x Ciud_Peq & 0,357 & 0,246 \\
\hline DifHosp x Numpers & $-0,103$ & 0,0587 \\
\hline DifPrecio x Cónyuge & $-0,034$ & 0,0334 \\
\hline DifPrecio x Salud1 & 0,118 & 0,104 \\
\hline DifPrecio x Salud2 & $-0,025$ & 0,0403 \\
\hline DifPrecio x Santiago_RM & 0,043 & 0,0528 \\
\hline DifPrecio x Ciud_Peq & $-0,026$ & 0,047 \\
\hline Constante & $-3,836^{\star \star \star}$ & 0,394 \\
\hline
\end{tabular}

Fuente: Elaboración propia.

Nota: $\quad{ }^{\star \star *}$ significativo al $1 \%$; ${ }^{* \star}$ significativo al $5 \%$; "significativo al $10 \%$.

\section{Efectos de la implementación del Plan Garantizado de Salud con prima comunitaria única}

Utilizando el modelo descrito en las secciones anteriores, se estima la transferencia de afiliados entre los sistemas de isapres y el FONASA luego de la implementación del PGS, con una prima comunitaria única. Para efectos de la simulación se corrigieron los datos proporcionados por la Comisión de Reforma de la Salud en lo relativo al nivel de cobertura del PGS. Se supuso un nivel de cobertura promedio de un $75 \%$, considerando los precios de 2013. Esto genera un valor de la tarifa plana de 26.989 pesos chilenos, por encima del rango estimado por la CRS, que consideraba coberturas más bajas (un 50\% en prestación ambulatoria FONASA libre elección y un 60\% en hospitalización en el Hospital Clínico de la Universidad de Chile José Joaquín Aguirre). Se consideró este aumento 
de la cobertura para acercarse a la propuesta realizada en la Comisión de Salud del Senado ${ }^{11}$. La propuesta de coberturas consideradas en el Senado a precios actuales implican un costo estimado por el Ministerio de Salud de tarifa plana de 28.432 pesos chilenos a pesos de 2013 por persona ${ }^{12}$. El valor de 26.989 pesos chilenos por beneficiario se obtuvo imponiendo la cobertura del $75 \%$ y calculando la prima que genera el mismo nivel de margen total del sistema de isapres que el que existía sin reforma. Este valor podría sobreestimar la prima comunitaria, si cae el margen total por efecto del aumento de la competencia que se podría generar al eliminar las preexistencias y carencias al interior del sistema de isapres. Finalmente, cabe señalar que el valor contempla un Subsidio por Incapacidad Laboral (SIL) del 1,5\% de la remuneración promedio de los cotizantes.

Los resultados obtenidos y mostrados en el cuadro 9 permiten concluir que un 12,39\% de la cartera total del sistema de isapres se transfiere al FONASA, aproximadamente un millón de personas. En todos los quintiles el efecto neto es la transferencia de las isapres al FONASA, no obstante son los grupos de menores ingresos los que se trasladan masivamente.

\section{Cuadro 9}

Chile: cambios en la cartera de isapres, por quintil (En porcentajes)

\begin{tabular}{lcc}
\hline Quintil de ingreso & $\begin{array}{c}\text { Variación cartera } \\
\text { de isapres }\end{array}$ & $\begin{array}{c}\text { Variación grupo } \\
\text { específico }\end{array}$ \\
\hline I & $-0,83$ & $-71,64$ \\
\hline II & $-2,41$ & $-53,10$ \\
\hline II & $-3,46$ & $-36,45$ \\
\hline IV & $-3,94$ & $-18,27$ \\
\hline V & $-1,76$ & $-2,78$ \\
\hline Total & $-12,39$ & $-12,39$ \\
\hline
\end{tabular}

Fuente: Elaboración propia, sobre la base de resultados de las regresiones y escenario simulado con el Plan Garantizado de Salud.

Si se compara la proporción de afiliados que se cambia por quintil de ingreso en relación con la participación inicial de cada grupo en las isapres, se advierte que el efecto de la reforma es acentuar la selección adversa en contra del FONASA. Solo el 1,76\% de las familias que se cambian pertenecen al $\checkmark$ quintil, mientras que ese grupo representa el $63,74 \%$ de los afiliados a isapres (véase el gráfico 1) ${ }^{13}$.

Las familias numerosas se cambian en mayor proporción que los individuos solteros o las familias pequeñas, como se observa en el cuadro 10. El 20,26\% de las familias de cuatro miembros o más se cambian al FONASA; este grupo representa el 15\% del total del sistema (véase el gráfico 2).

Esto también representa un efecto de selección adversa que genera un aumento del déficit del FONASA, toda vez que en el FONASA las familias hacen un aporte proporcional al ingreso (7\%) pero el gasto depende del número de cargas.

\footnotetext{
${ }^{11}$ La propuesta del Ministerio considera una cobertura en hospitalización del 80\% (arancel hospital J. J. Aguirre) y del 60\% ambulatoria, elevando la cobertura establecida en el estudio del Consejo de Reforma de la Salud.

12 Informe del Ministro de Salud a la Comisión de Salud del Senado, junio de 2013.

${ }^{13}$ Están sobrerrepresentadas las familias de menores ingresos entre los que emigran al FONASA, en relación con la población promedio de las isapres.
} 


\section{Gráfico 1}

Chile: participación en las isapres, por quintil de ingreso, 2013

(En porcentajes)

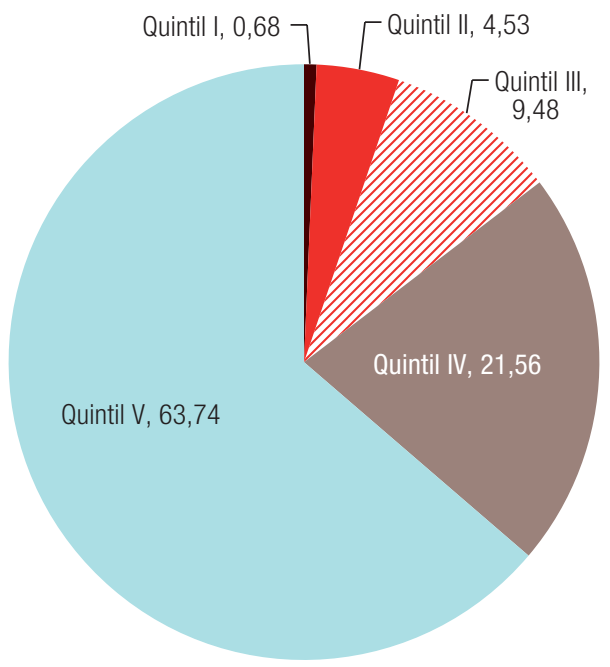

Fuente: Elaboración propia, sobre la base de datos de la Encuesta de Caracterización Socioeconómica Nacional (CASEN) 2013.

Cuadro 10

Chile: cambios en la cartera de isapres, por grupo familiar (En porcentajes)

\begin{tabular}{lcc}
\hline Núcleo familiar & $\begin{array}{c}\text { Variación cartera } \\
\text { de isapres }\end{array}$ & $\begin{array}{c}\text { Variación grupo } \\
\text { específico }\end{array}$ \\
\hline 1 persona & $-1,96$ & $-5,69$ \\
\hline 2 personas & $-3,44$ & $-12,34$ \\
\hline 3 personas & $-3,87$ & $-17,42$ \\
\hline 4 personas o más & $-3,12$ & $-20,26$ \\
\hline Total & $-12,39$ & $-12,39$ \\
\hline
\end{tabular}

Fuente: Elaboración propia, sobre la base de resultados de las regresiones y escenario simulado con el Plan Garantizado de Salud.

\section{Gráfico 2}

Chile: participación en las isapres, por grupo familiar, 2013 (En porcentajes)

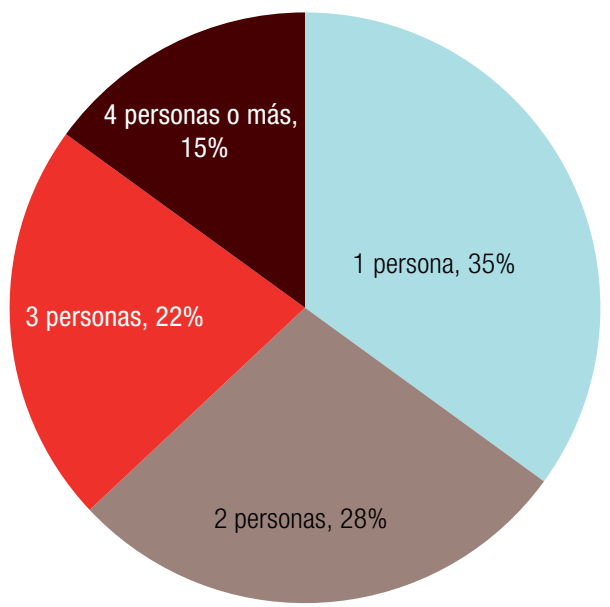

Fuente: Elaboración propia, sobre la base de datos de la Encuesta de Caracterización Socioeconómica Nacional (CASEN) 2013. 
Respecto a las condiciones de salud de los que emigran al FONASA, como se observa en el cuadro 11, no sería un factor significativo que genere selección adversa (véase además el gráfico 3).

\section{Cuadro 11}

Chile: cambios en la cartera de isapres, por estado de salud (En porcentajes)

\begin{tabular}{lcc}
\hline Estado de salud & $\begin{array}{c}\text { Variación cartera } \\
\text { de isapres }\end{array}$ & $\begin{array}{c}\text { Variación grupo } \\
\text { específico }\end{array}$ \\
\hline Bueno & $-9,69$ & $-10,52$ \\
\hline Razonable & $-3,09$ & $-46,83$ \\
\hline Malo & 0,40 & 33,04 \\
\hline Total & $-12,39$ & $-12,39$ \\
\hline
\end{tabular}

Fuente: Elaboración propia, sobre la base de resultados de las regresiones y escenario simulado con el Plan Garantizado de Salud.

\section{Gráfico 3}

Chile: participación en las isapres, por estado de salud, 2013 (En porcentajes)

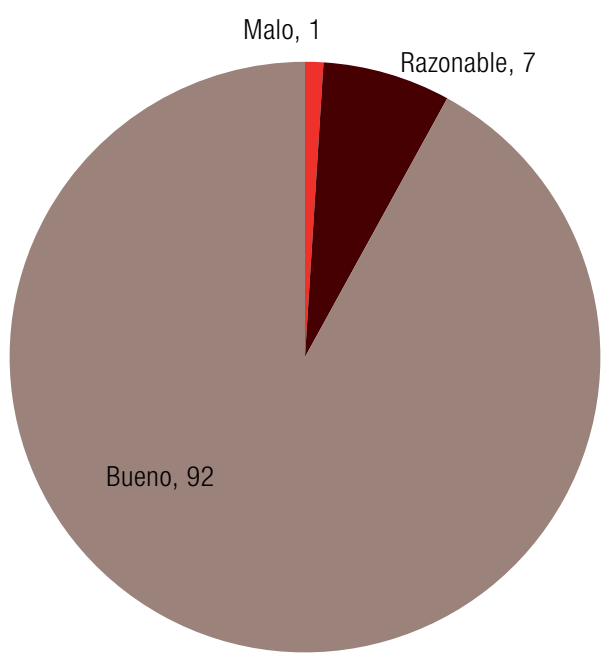

Fuente: Elaboración propia, sobre la base de datos de la Encuesta de Caracterización Socioeconómica Nacional (CASEN) 2013.

Finalmente, para efectos de simular el costo para el FONASA de los 400.000 beneficiarios desde las isapres, consideramos la información disponible hasta 2011 sobre costos para el FONASA grupo D como una estimación del costo de atención, ajustada por el IPC de salud hasta 2013 y considerando un costo para el FONASA de 26.190 pesos chilenos ${ }^{14}$.

Como en el FONASA se cobra el $7 \%$ del ingreso, con el cual se debe financiar el Subsidio por Incapacidad Laboral por enfermedad (1,5\% del ingreso), el ingreso de los que se cambian en promedio es de 251.003 pesos chilenos y el número promedio de miembros en la familia es 2,9, de manera que el aporte promedio por beneficiario es de 5.522 pesos chilenos por mes. El déficit del FONASA por cada persona que se cambia sería de 20.668 pesos chilenos al mes. Considerando la situación de precios de 2013, el déficit anual por beneficiario transferido sería de 248.015 pesos chilenos, monto similar al gasto total del FONASA por beneficiario promedio. En conclusión, la reforma implica aumentar un 12\% el gasto del FONASA para atender a los beneficiarios que se trasladan al sistema público.

\footnotetext{
14 Véase Bitran, Arpón y Debrott (2013).
} 


\section{Conclusiones}

En el presente estudio se desarrolla un modelo de elección discreta, utilizando datos de la encuesta CASEN 2013, para modelar la elección de seguro de salud de los cotizantes en Chile. A diferencia de estudios anteriores, el modelo deja de usar el factor de riesgo individual como variable sustitutiva del precio del seguro privado y utiliza un vector de precios estimados de acuerdo con la edad, el número de cargas y el nivel de ingreso del cotizante. Por otro lado, se considera explícitamente en el modelo la influencia de la calidad de los sistemas en la elección del seguro de salud, lo cual, como vemos en nuestro análisis, mejora la capacidad explicativa del modelo.

Este modelo se usa para simular cómo cambiaría la elección de los cotizantes al introducir el PGS, con prima única. Es decir, para simular cambios en la demanda de planes de salud, de manera que los resultados muestran un análisis parcial del efecto del PGS. Este efecto parcial no considera la posible reacción por parte de la oferta en el sentido de agregar planes complementarios o suplementarios al PGS. Estos posibles nuevos planes cambiarían por un lado la "prima total" y la "cobertura total" que los individuos enfrentarían, lo que cambiaría la demanda de planes de salud. Por ejemplo, si un individuo tiene preferencias fuertes por cobertura, pero también por precio, al enfrentar dos planes similares en esas condiciones no sería claro cuál elegiría. Sin embargo, si a este mismo individuo le agregamos un plan que complementa el plan básico que tiene a una prima aceptable, este individuo elegiría este plan.

Sin embargo, este efecto parcial por el lado de la demanda es suficientemente grande como para no considerarlo dentro de un análisis más exhaustivo de la oferta y demanda de seguros privados.

Nuestros resultados indican que la cartera de isapres disminuiría un 12,39\%, lo que significa que alrededor de 400.000 personas se cambiarían al FONASA ${ }^{15}$. Esta disminución se compone principalmente de familias numerosas y personas pertenecientes a quintiles de ingresos más bajos. Este resultado exacerba los problemas de selección adversa presentes en el sistema FONASA. Es decir, la introducción del PGS tendría un impacto financiero adverso en el FONASA, disminuyendo la capacidad de este de cumplir con sus obligaciones.

Como vimos, la reforma implica aumentar un 12\% el gasto del FONASA para atender a los beneficiarios que se trasladan al sistema público. Considerando el presupuesto del año 2013, se estima que el costo de la implementación del PGS con tarifa plana sería de alrededor de 200 millones de dólares al año para el fisco. Además del estrés financiero que implica un aumento inmediato del gasto de esta magnitud, el FONASA deberá ser capaz de incorporar unas 400.000 personas que están acostumbradas a una modalidad de servicio diferente, con todas las dificultades que esto conlleva. Estas familias probablemente gastarán más que el promedio de los cotizantes del grupo D del FONASA, ya que están acostumbradas a la Modalidad Libre Elección. Por otro lado, este cambio masivo produciría en alguna medida un desplazamiento de la población actual del FONASA en el uso de recursos, ya que tiene menor entrenamiento en el uso de seguros y menores recursos.

En conclusión, no es conveniente implementar un Plan Garantizado de Salud con prima comunitaria única, en un sistema de seguros de salud tan segmentado como el chileno, toda vez que tiende a exacerbar el problema de selección adversa ya presente en el sistema, acrecentando la segmentación y requiriendo una enorme cantidad de recursos adicionales del Estado. En este contexto, se recomienda analizar una política de precios más solidaria para el PGS, considerando de manera diferenciada e integral (sistema público y privado) la demanda, apuntando a distintas primas de acuerdo con factores como la edad y el nivel socioeconómico de los cotizantes y la interacción con la oferta.

\footnotetext{
${ }^{15}$ La simulación se hace sobre la base de dos millones de cotizantes, que representan un porcentaje importante de la cartera total. Esto es necesario debido a las restricciones de datos que tenemos al usar encuestas. En particular la base de datos excluye a los cotizantes independientes y por lo tanto podría introducir sesgo.
} 


\section{Bibliografía}

Akerlof, G. A. (1970), "The market for 'lemons': quality uncertainty and the market mechanism", The Quarterly Journal of Economics, vol. 84, $\mathrm{N}^{\circ} 3$.

Bitran, R., P. Arpón y D. Debrott (2013), "Opciones de reforma a la seguridad social en salud en Chile", Documento de Referencia, №13, Santiago, Espacio Público.

Copetta, C. (2011), "Minuta Plan Garantizado de Salud Para el Sector Isapres", Documento de Trabajo, Santiago, Superintendencia de Salud.

Cutler, D. M. y S. J. Reber (1998), "Paying for health insurance: the trade-off between competition and adverse selection", The Quarterly Journal of Economics, vol. 113, № 2, Oxford, Oxford University Press.

Dawes Ibáñez, A. (2010), "Health care reform and its effect on the choice between public and private health insurance: evidence from Chile", tesis, Santiago, Instituto de Economía, Pontificia Universidad Católica de Chile.

Duarte, F. (2012), "Price elasticity of expenditure across health care services", Journal of Health Economics, vol. 31, № 6, Amsterdam, Elsevier.

Feldman, R. y otros (1989), "The demand for employment-based health insurance plans", Journal of Human Resources, vol. 24, № 1, Wisconsin, University of Wisconsin Press.

Friedman, B. (1974), "Risk aversion and the consumer choice of health insurance option", The Review of Economics and Statistics, vol. 56, № 2, Cambridge, Massachusetts, The MIT Press.

Missoni, E. y G. Solimano (2010), "Towards universal health coverage: the Chilean experience", World Health Report (2010) Background Paper, № 4, Organización Mundial de la Salud (OMS) [en línea] https://pdfs.semanticscholar. org/226c/4621 cd397092dfd556f7b19f02048d6f5620.pdf?_ga=2.163282104.523300194.15019662371570332244.1501185329.

Sanhueza, R. y J. Ruiz-Tagle (2002), "Choosing health insurance in a dual health care system: the Chilean case", Journal of Applied Economics, № 001.

Sapelli, C. y A. Torche (2001), "The mandatory health insurance system in Chile: explaining the choice between public and private insurance", International Journal of Health Care Finance and Economics, vol. 1, N ${ }^{\circ} 2$, Springer.

(1998), "El seguro previsional de salud: determinantes de la elección entre seguro público y privado, 1990-1994", Cuadernos de Economía, vol. 35, № 106, Santiago, Instituto de Economía, Pontificia Universidad Católica de Chile.

Sapelli, C. y B. Vial (2003), "Self-selection and moral hazard in Chilean health insurance", Journal of Health Economics, vol. 22, № 3, Amsterdam, Elsevier.

Superintendencia de Salud (2012), "Estudio de opinión y satisfacción de usuarios", Santiago [en línea] http:// www.supersalud.gob.cl/documentacion/666/articles-7632_recurso_1.pdf.

Tokman, M., C. Marshall y C. Espinosa (2007), "Un matrimonio difícil: la convivencia entre un seguro público solidario y seguros de salud competitivos", serie Financiamiento del Desarrollo, № 199 (LC/L.2851-P), Santiago, Comisión Económica para América Latina y el Caribe (CEPAL). 